\title{
COMPARACIÓN DE LA INDUSTRIA TURÍSTICA EN POTENCIAS EMERGENTES (ARGENTINA Y BRASIL): DE LA RIVALIDAD A LA INTERDEPENDENCIA
}

\author{
Marco Garrido Cumbrera \\ Carolina A. Fernández de León \\ Olta Braçe \\ Universidad de Sevilla
}

\section{RESUMEN}

En los últimos años, además de los destinos maduros de Europa y América del Norte, han irrumpido con fuerza nuevos subcontinentes como América del Sur. La industria turística de Argentina y Brasil ha evolucionado favorablemente, mostrando un considerable crecimiento y estabilidad económica que les ha convertido en potencias turísticas emergentes. En este artículo se presenta, de manera comparada, la evolución del sector turístico en ambos países, identificando las principales causas que han influido en su desarrollo. El interés de esta comparación radica en que ambos países presentan importantes atractivos turísticos, fuerte crecimiento económico, grandes desequilibrios territoriales y altos niveles de inseguridad, unidos a una compleja interdependencia en cuanto a los flujos turísticos. Comprender estas interrelaciones resulta vital para profundizar en los mecanismos de cooperación e intercambio, permitiendo acabar definitivamente con los conflictos y rivalidades.

Palabras clave: Turismo; Potencias emergentes; Desarrollo económico; Argentina y Brasil.

Recibido: 24 de junio de 2015

Devuelto para su revisión: 14 de octubre de 2015

Aceptado: 20 de noviembre de 2015

Departamento de Geografía Física y Análisis Geográfico Regional. Universidad de Sevilla. C/ Doña María de Padilla, s/n. 41004 SEVILLA (España).E-mail: mcumbrera@us.es 


\title{
A comparison of tourism industry in emerging powers (Argentina and Brazil): from rivalry to interdependence
}

\begin{abstract}
Over the past few years, in addition to the mature destinations in Europe and North America, new regions such as South America have burst onto the scene. The tourism industry in Argentina and Brazil has developed positively, showing significant growth and economic stability that has made them emerging tourist powers. This article presents, in a comparative way, the historical development of tourism in both countries, identifying the main causes that have influenced its development. The importance of this comparison lies in the fact that both countries have important tourist attractions, strong economic growth, large regional inequalities and high levels of insecurity, together with a complex competitive interdependence. Understanding these relationships is vital to delve into the cooperation and exchange mechanisms that can definitively end the conflict and rivalries.
\end{abstract}

Keywords: Tourism; Emerging powers; Economic Development; Argentina and Brazil.

\section{INTRODUCCIÓN}

\subsection{Características generales de Argentina y Brasil}

Argentina y Brasil son los dos países más extensos de América del Sur (2,8 y 8,5 millones de $\mathrm{km}^{2}$ respectivamente) y de los más poblados (41,5 y 200,4 millones de habitantes).

Ambos países representan las principales economías de América del Sur y forman parte de MERCOSUR y UNASUR a nivel continental y a nivel mundial del G20 lo que las convierte en grandes potencias emergentes (Orgaz y cols., 2011). El Banco Mundial clasifica a Argentina y Brasil como países de ingresos medios altos y la CEPAL cifra su PIB anual (2013) en los 524.030 y los 2.279 .748 millones de dólares respectivamente. El Programa de las Naciones Unidas para el Desarrollo, que elabora el Índice de Desarrollo Humano (IDH), clasifica a Argentina dentro del grupo de desarrollo humano muy alto (posición 45) y a Brasil con desarrollo humano alto (posición 85) (PNUD, 2012).

Figura 1

DATOS BÁSICOS DE ARGENTINA Y BRASIL

\begin{tabular}{|l|c|c|}
\hline & Argentina & Brasil \\
\hline Población $(2013)^{1}$ & 41.45 millones & 200.4 millones \\
\hline PIB (US\$) $2013^{2}$ en millones de dólares & $524.030 \$$ & $2.279 .748 \$$ \\
\hline GDP per capita 2011-2015 (current US\$) & 12.509 & 11.727 \\
\hline Clasificación según Ingresos 2014 $^{1}$ & Ingresos Medios Altos & Ingresos Medios Altos \\
\hline${\text { Esperanza de vida al nacer, total (años) } 2012^{1}}^{\text {Índice de Desarrollo Humano - Ranking }}{ }^{3}$ & 76 & 74 \\
\hline
\end{tabular}

Fuente: Banco Mundial, 2014'; CEPAL, 2013², UNDP, $2013^{3}$. 


\subsection{Principales destinos turísticos en Argentina y Brasil}

Para entender la posición de Argentina y Brasil como las principales potencias turísticas de América del Sur resulta necesario señalar algunos de los atractivos y destinos turísticos más significativos y visitados en ambos países.

Brasil posee la industria turística más diversificada del subcontinente, contando con una población acogedora, la selva amazónica como principal atractivo natural del mundo, culturas diversas y únicas, miles de kilómetros de playas vírgenes o grandes superficies de humedales (Santana, 2000). Sin embargo, Brasil presenta una imagen bien definida, basada en sus playas, su cultura musical, el carnaval de Río de Janeiro y de Salvador o la gran diversidad cultural. En efecto, el brasileño es el resultado de la mezcla de las culturas portuguesa, italiana, española, alemana, japonesa, de Europa del Este y africana, cuya variedad ha influido en formas de expresión culturales relacionadas con las artes, la música, las normas sociales o el comportamiento (Crane \& Rizowy, 2011). Por todo ello, Brasil es el país de América del Sur que concentra más lugares declarados Patrimonio de la Humanidad por la UNESCO, con ciudades históricas como Salvador de Bahia, Ouro Preto, Olinda y Goiás, que atesoran un rico pasado colonial.

La marca turística Brasil se relaciona con la exuberancia, el mestizaje, la hospitalidad o la alegría, constituyendo uno de sus principales activos para la promoción turística (Álvarez de la Torre y Rodríguez-Toubes Muñiz, 2013). En efecto, entre los principales esterotipos de Brasil destaca el «tropicalismo» el cual engloba recursos turísticos como la alegría de sus gentes, el exotismo, las playas, carnaval, etc.; aunque igualmente encierra la idea de peligrosidad, riesgo, delincuencia o incluso cierta dosis de machismo (Ribeiro, 2002).

Si nos centramos en el número de llegadas internacionales que visitaron Brasil en 2013 destaca principalmente la ciudad de São Paulo con un total de 2.219.513 llegadas, seguida de Río de Janeiro con 1.207.800 turistas (Ministerio de Turismo de Brasil, 2013). Precisamente, la mayor parte de las actividades económicas de Brasil se concentran en el triángulo que forman las ciudades de Río de Janeiro y São Paulo, junto a Belo Horizonte (en el estado de Minas Gerais), mientras que otros estados, sobre todo del Noreste, registran bajas rentas per cápita, constatándose importantes desequilibrios inter-estatales. No es de extrañar que el mayor número de ofertas de alojamiento se concentren en las ciudades de Río de Janeiro y São Paulo. Aunque el mayor número de camas se encuentran en São Paulo, Río de Janeiro concentra el mayor número de establecimientos (778 Río de Janeiro y 769 São Paulo) (Ministerio de Turismo de Brasil, 2014).

Entre los destinos naturales del mundo destaca el Amazonas como el mayor bosque tropical del mundo, cuya extensión lo convierten en el mayor Estado de Brasil (Ribeiro y Nelson, 2014).

Tradicionalmente Argentina ha mirado hacia Europa y no al resto de América del Sur en el comercio y la cultura, desarrollando una gran actividad económica que -antes de la Segunda Guerra Mundial- la convirtió en uno de los países más ricos del mundo gracias a la libre circulación de capitales y a la estabilidad monetaria. Sin embargo, gran parte de esta riqueza se desperdició como resultado de proyectos económicos fallidos y décadas de luchas políticas. Uno de los principales problemas del país es el escaso 
desarrollo de sus infraestructuras de transporte. Aunque los servicios aéreos internos han mejorado, no ocurre lo mismo con su red de carreteras y ferrocarriles que, construidas a principios del siglo $\mathrm{XX}$, han experimentado escasas mejoras.

El documento «Visión de la Argentina», realizado por el Departamento de Turismo de la Administración General de Parques Nacionales y Turismo (1950), destacaba los paisajes naturales, los edificios históricos y el folclóre como los principales atractivos turísticos de Argentina. Esta variedad de destinos de Argentina se resumía en la siguiente afirmación: «si en los lagos del sur el turismo es deporte, en el norte descanso, en la Patagonia vastedad, en Córdoba es salud» (AGPNyT, 1950). Actualmente, de acuerdo a los indicadores registrados en 2013 por el Ministerio de Turismo de Argentina (2014), las regiones que registran un mayor volumen de visitantes son las de Buenos Aires, Litoral, Córdoba y Norte. Bertoncello (2006) detalla los principales destinos en cada una de las regiones comenzando con la región noroeste en donde cita Quebrada de Humahuaca y las ciudades de Salta y Tucumán; en el Nordeste, las Cataratas del Iguazú y la ciudad de Mendoza. En la región centro, las sierras de Córdoba y en la región pampeana, Mar del Plata y la ciudad de Buenos Aires; mientrás en la región Patagónica los parques nacionales como el de Nahuel Huapi y la ciudad de Bariloche. En el Área Natural Protegida Punta Tombo ubicado en la Patagonia se concentra, entre los meses de septiembre y abril, la mayor colonia continental de pingüinos de Magallanes del mundo. Mar del Plata supone el destino de sol y playas más importante de la costa atlántica, cuya separación de poco más de $400 \mathrm{~km}$. respecto a Buenos Aires la convierten en el principal destino turístico de los bonaerenses (Sánchez y Kaczan, 2013). Son muchos los brasileños que durante la temporada de nieve visitan los centros de ski de Argentina (específicamente Bariloche), que compiten con los centros chilenos por atraer turistas brasileños. Por su parte, las Cataratas del Iguazú, declarado Patrimonio Natural de la Humanidad en 1984, supone un importante destino turístico situado en la triple frontera entre Argentina, Brasil y Paraguay.

\section{EVOLUCIÓN HISTÓRICA DEL TURISMO EN ARGENTINA Y BRASIL}

Si analizamos la evolución histórica del turismo en Argentina y Brasil, se observa como a principios de la década de los setenta el número de turistas internacionales que llegaban a Argentina era considerablemente superior al de Brasil. Esta diferencia continuó aumentando, llegando a alcanzar Argentina la cifra de 1.350.000 visitantes en 1977, mientras Brasil permanecía por debajo con los 635.000 turistas (OMT, 1979). A pesar del importante volumen de turistas, hasta la década de los 80 el turismo no adquirió gran relevancia en Argentina en términos económicos. Sin embargo, debido al colapso económico sufrido, el turismo se convertiría en una importante fuente de ingresos de divisas y de creación de empleo. La industria turística argentina, que se había orientado principalmente al mercado interno, comenzó a promocionar sus destinos en el mercado internacional. De este modo, los empresarios vinculados al sector intentaron añadir, a la corriente de turistas procedente de países limítrofes, visitantes de Europa y Norteamérica (Goded y Parache, 2006). 
Por su parte, los años 70 y 80 significaron para Brasil una época de crecimiento en la llegada de turistas internacionales aunque ese crecimiento generalizado fue acompañado de importantes descensos en determinados años. Así, si en 1989 se registraron 1.402.879 turistas extranjeros, al año siguiente (1990) se alcanzaron cifras similares a las de 1979 (1.081.799 turistas internacionales). Afortunadamente dicho periodo de retroceso sería superado y, a partir de 1991, el turismo internacional volvería a crecer en Brasil.

\subsection{Década de los 90}

Durante la década de los noventa, América del Sur se convertiría en un importante destino turístico, aumentando el número de turistas internacionales en su conjunto con Brasil, Argentina y Chile como los principales países receptores. Durante el quinquenio 1990-95, se produjeron tasas de crecimiento por encima del 150\%, en el caso de Argentina, y del $80 \%$, en el caso de Brasil.

Es necesario señalar como la evolución del sector turístico de los países de América del Sur ha estado estrechamente ligada al desarrollo de sus economías y a las crisis financieras sufridas. Así, la recesión económica brasileña de 1998 tuvo repercusiones tanto en el poder adquisitivo de los brasileños como en el coste de vida (OMT, 2014a). De este modo, el colapso de la economía brasileña lo convertiría en un país barato para visitar, aumentando en más de 2 millones las llegadas internacionales en tan solo un año, hasta lograr los 5,3 millones en el año 2000. Por su parte, Argentina que había comenzado la década de los 90 con un número de llegadas internacionales que casi duplicaban a las de Brasil, durante dicha década crecería de manera más moderada, quedando, al final de la década, con menos de 3 millones respecto a los más de 5 millones de Brasil.

\section{Figura 2 \\ EVOLUCIÓN DE LA LLEGADA DE TURISTAS INTERNACIONALES A ARGENTINA Y A BRASIL (1990-2000)}

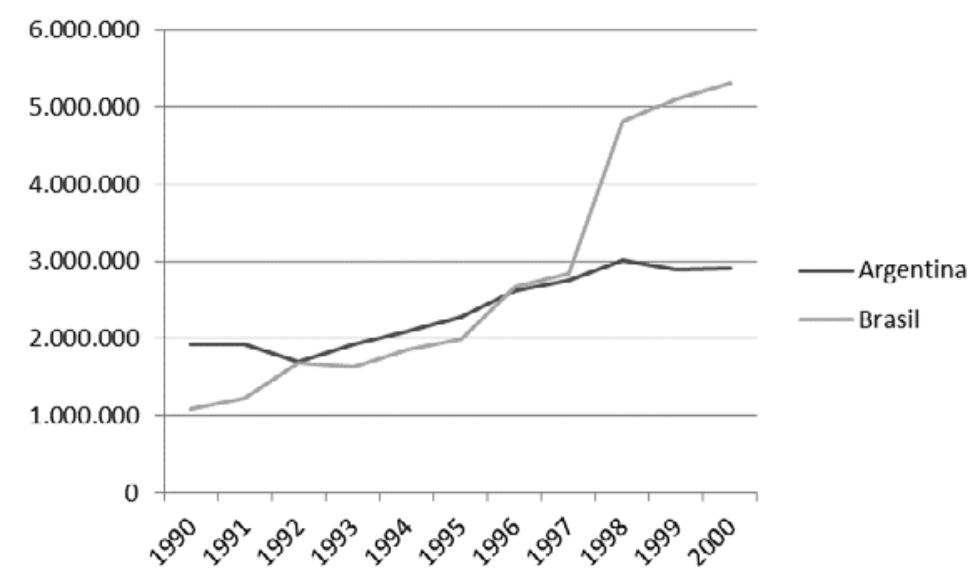

Fuente: elaboración propia a partir de datos de los ministerios de turismo de Brasil y de Argentina. 


\subsection{Década 2000-2010}

Entre 1998 y 2002 se produciría una reducción del 18\% en el número de turistas internacionales en América del Sur debido a factores externos como el conflicto de Iraq y los continuos miedos tras el 11 de septiembre de 2001, unido a la inestabilidad política y económica de algunos de los países del subcontinente como Argentina (Sharma, 2008) o Brasil (Sobral et al., 2007). En los primeros 5 años del siglo XXI se produjo una caída de la contribución de Argentina al mercado turístico de América del Sur con un descenso del 40\% en 2000, al 22\% entre 2002-2005 (Mintel, 2006). La caída del flujo de turistas hacía Argentina está relacionada con el colapso de su economía a partir del 1 de diciembre de 2001 («corralito bancario»), que impuso restricciones a la retirada de dinero de los bancos y redujo drásticamente el poder adquisitivo de los argentinos. Dicha crisis produjo graves efectos en la economía argentina, desmantelando su clase media y disminuyendo el número de llegadas internacionales. En 2002 Argentina alcanzó niveles de pobreza históricos, con tasas de pobreza que superaban el 55\%, afectando a casi 21 millones de personas (Haverland, 2008). A finales de ese mismo año, Argentina se encontraba en situación de incumplimiento de sus obligaciones de pago y Brasil tenía cerrado el mercado para nuevas colocaciones (Frenkel, 2003).

Según datos proporcionados por los respectivos Ministerios de Turismo, coincidiendo con el periodo de recesión económica mundial de 2008, Argentina y Brasil registraron un importante descenso respecto al nivel de ingresos y de turistas internacionales. Sin embargo, a partir de 2009, tanto los ingresos como las llegadas de turistas han continuado en ascenso hasta 2011, año en que se produce un nuevo estancamiento de los ingresos tanto en Brasil como en Argentina.

\subsection{Recientemente y en la actualidad}

Según las estadísticas de los Ministerios de Turismo de Brasil y de Argentina de 2013, la llegada de turistas internacionales a Brasil alcanzó los 5.813.342, mientras que en Argentina ascendió a 5.570.969. Argentina y Brasil continúan constituyendo los principales destinos turísticos de América del Sur, con una cuota del 20,7\% en Brasil y del 20,4\% en Argentina, seguidos de Chile (13\%) y Perú $(11,5 \%)$ en 2013. Ello significa que Brasil y Argentina juntos reciben más del $40 \%$ del total de turistas internacionales de América del Sur. De los ingresos generados por el sector turístico en América del Sur durante 2013, el $46 \%$ se concentraba en Argentina y Brasil (un 18\% Argentina y un 28\% Brasil), siendo la estancia media de los turistas internacionales de 11,3 noches en Argentina, frente a las 17,3 noches en Brasil.

Resulta interesante poner de relieve el papel que ha tenido MERCOSUR como proceso de integración de América del Sur -Argentina, Brasil, Paraguay, Uruguay, Venezuela y Bolivia-, traduciéndose en un aumento de las transacciones con consecuencias positivas en el desarrollo del sector turístico tanto de Brasil como de Argentina (Goded y Parache, 2006).

El estancamiento de la economía argentina producido a finales de 2013 unido a la desaceleración registrada durante el primer cuatrimestre de 2014 ha hecho caer tanto las 
exportaciones como las importaciones. Entre las medidas adoptadas por el gobierno argentino se incluyen devaluaciones del peso que han servido para aumentar la competitividad del sector turístico argentino.

Figura 3

\section{EVOLUCIÓN DE LA LLEGADA DE TURISTAS INTERNACIONALES A ARGENTINA Y A BRASIL (2000-2013)}

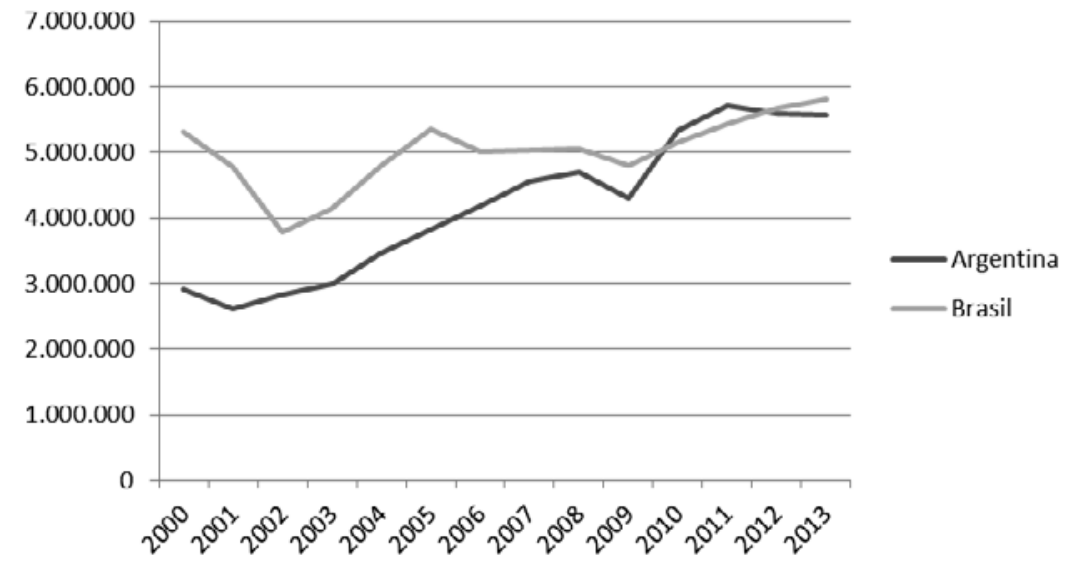

Fuente: elaboración propia a partir de datos de los Ministerios de Turismo de Brasil y de Argentina.

Los gastos en turismo realizados por Brasil son relativamente superiores a los realizados por Argentina debido principalmente a la mayor cantidad de establecimientos y plazas de alojamiento turístico y a las mayores inversiones realizadas en los últimos años. Así en 2012, Brasil ocupaba el puesto decimosegundo del mundo en lo referente a los gastos producidos por el turismo con 22.200 millones de dólares EEUU (OMT, 2013a).

En cuanto a los ingresos por turismo registrado en ambos países, Brasil se encuentra a la cabeza con 6.843 millones de dólares EEUU, frente a los 4.627 millones de dólares de Argentina registrados en 2014 (OMT, 2015).

\section{RELACIONES ENTRE EL TURISMO DE BRASIL Y DE ARGENTINA}

La economía y los sectores turísticos de Argentina y de Brasil están conectados y son interdependientes. Así, durante muchos años, Argentina ha sido el país de procedencia más importante para Brasil, aunque se hayan producido fluctuaciones dependientes de la situación económica y del valor del peso argentino (Prideaux and Carson, 2010). Así, en 1995 el turismo internacional de Brasil estaba compuesto por un 33\% de argentinos, manteniéndose estable durante el año 2000 (Gardella y Aguayo, 2006). Debido a la crisis económica que sufrió Argentina en 2002, su participación cayó del 33\% en 2000 al 19\% en 2004. Sin embargo, actualmente Argentina sigue ocupando el primer lugar de procedencia de los turistas que visitan Brasil, con una cuota que alcanza el 29\% del total de llegadas 
registradas en Brasil en 2013 (1.711.491), colocándose como primer país de procedencia. Entre 2003 y 2013 el número de turistas argentinos que visitaron Brasil aumentó un 102,9\%. Los argentinos visitan principalmente otros países limítrofes como Uruguay y Chile, siendo el principal país de origen (Brida, Lanzilotta, Risso, 2008), alcanzando el 41,1\% del total de las llegadas en el caso de Chile en 2013 (SERNATUR, 2014).

Por su parte, Brasil, que en los últimos años viene siendo el principal impulsor del crecimiento del turismo receptor en Argentina, se encuentra en medio de un proceso de desaceleración económica produciendo disminuciones tanto en la cantidad de turistas como en el gasto medio (Ministerio de Turismo de Argentina, 2014; Ministerio de Turismo de Brasil, 2014). Del mismo modo, Brasil fue el país que más gasto realizó en Argentina en 2013, suponiendo el 20,4\% de los ingresos totales. La llegada de brasileños a Argentina constituyó un 19\% del total de llegadas internacionales (1.083.250 en 2013), lo que supuso un descenso respecto al año anterior (1.217.374 en 2012), contribuyendo al descenso del turismo internacional. Por lo tanto, se puede afirmar que la reducción del número de turistas brasileños que visitaron Argentina tuvo una fuerte repercusión en la caída de los ingresos por turismo internacional registrada en Argentina. De hecho, Brasil fue responsable de más de $2 / 3$ partes de la caída del turismo internacional que experimentó Argentina. De este modo en 2013 Chile consiguió superar a Brasil en cuanto a las llegadas a Argentina, convirtiéndose en el principal mercado emisor de Argentina (Ministerio de Turismo de Argentina, 2014; Ministerio de Turismo de Brasil, 2014).

\section{MEGA-EVENTOS DEPORTIVOS}

En los últimos años, debido a las celebraciones de la Copa del Mundo de Fútbol en 2014 y los Juegos Olímpicos de Río de Janeiro 2016 se han producido, y aún siguen realizándose, importantes inversiones en infraestructuras, preparación y organización de eventos, aumentando considerablemente el número de empleados en el sector turístico. Ello ha permitido un aumento considerable tanto de la oferta de establecimientos turísticos, como de las plazas disponibles, promocionando Brasil como uno de los principales destinos turísticos internacionales.

Sin embargo, la imagen de inseguridad que se proyecta del destino Brasil contribuye a aumentar la percepción de riesgo (Álvarez de la Torre y Rodríguez-Toubes Muñiz, 2013). En efecto, la planificación turística de Brasil (2010-2014) identifica la inseguridad como una de las debilidades a superar, principalmente en las ciudades sede de los eventos (Ministerio de Turismo de Brasil, 2010).

La organización de estos dos mega-eventos deportivos de alcance internacional durante un período de tan sólo dos años, supone un auténtico desafío debido a la existencia de importantes conflictos sociales como la delincuencia y la marginalidad, consecuencia de las desigualdades sociales existentes.

Debido a la gran capacidad competitiva, derivada de sus menores costes y sus atractivos turísticos, las proyecciones de futuro muestran como a medio plazo ambos países pasarán a convertirse en potencias estratégicas dentro del nuevo panorama de un mundo cada vez más conectado. En este sentido, el informe de la OMT «The Tourism 2020 Vision» estima que la evolución futura de la llegada de turistas internacionales al conti- 
nente americano llegará a alcanzar los 232 millones en 2020, con aumentos en el número de llegadas internacionales del 5\% anual tanto en Argentina como en Brasil (OMT, 2014a, OMT, 2014b).

\section{CONCLUSIONES}

El análisis comparado de la situación del sector turístico de Argentina y de Brasil nos ha permitido extraer una serie de conclusiones importantes para comprender la evolución temporal y las interrelaciones existentes en el seno de América del Sur, analizando las dos principales potencias turísticas del subcontinente.

Se ha puesto de manifiesto, el alto nivel de interdependencia turística de ambos países debido a que, como se ha analizado, un elevado porcentaje de las llegadas internacionales son argentinos, en el caso de Brasil, y brasileños, en el caso de Argentina. A pesar de que Argentina y Brasil son dos países fronterizos que necesitan cooperar, la realidad muestra como compiten por atraer al mayor número de turistas internacionales.

En los últimos años, ambos países han mostrado un considerable crecimiento y mejora en la estabilidad económica que ha realzado su papel como importantes destinos turísticos internacionales. Por ello, el sector turístico, tanto de Brasil como de Argentina, ha evolucionado positivamente, salvo durante los años de recesión económica (2001 y 2008).

Los acontecimientos deportivos de alcance mundial de Brasil han propiciado una tendencia positiva respecto al número de llegadas internacionales. Argentina, por el contrario, deberá sobreponerse al descenso de llegadas causado en gran medida por la reducción de los turistas brasileños. En lugar de enfatizar las diferencias entre sus respectivos destinos turísticos, ambos países deberán resolver sus discrepancias y aprovechar la creciente interdependencia de sus economías y las sinergias de sus sectores turísticos para así hacer frente a amenazas externas. Afianzar los lazos de unión y las relaciones bilaterales, basadas en la interdependencia, resultan vitales para cerrar antiguas rivalidades y continuar desarrollando sus respectivos sectores turísticos en el seno de MERCOSUR y UNASUR.

\section{BIBLIOGRAFÍA}

ADMINISTRACIÓN GENERAL DE PARQUES NACIONALES Y TURISMO (AGPNyT). (1950): Ministerio de Obras Públicas. Visión de Argentina 1950. Buenos Aires: Peuser.

ÁLVAREZ DE LA TORRE, J. y RODRÍGUEZ-TOUBES MUÑIZ D. (2013): «Riesgo y percepción en el desarrollo de la imagen turística de Brasil ante los mega-eventos deportivos», Pasos. Revista de Turismo y Patrimonio Cultural, vol. 11, n 3, pp. 147154.

BANCO MUNDIAL [en línea] URL disponible en: http://datos.bancomundial.org/indicador/NY.GDP.MKTP.KD.ZG (Último acceso 19 Mayo 2014).

BERTONCELLO, R. (2006): «Turismo, territorio y sociedad. El mapa turístico de la Argentina». En: Geraiges de Lemos A.I., Arroyo M., Silveira M.L. América Latina: cidade, campo e turismo. San Pablo: Consejo Latinoamericano de Ciencias Sociales. 
BRIDA J.G., LANZILOTTA B. y RISSO W.A. (2008): «Turismo y crecimiento económico: el caso de Uruguay», Pasos. Revista de Turismo y Patrimonio Cultural, vol. 6 (3), pp 481-492.

CRANE, R., RIZOWY, C. (2011): «Latin American Business Cultures». London: Palgrane Macmillan.

FRENKEL, R. (2003): «Globalización y crisis financieras en América Latina», Revista de la Cepal, vol. 80. Agosto 2013.

GARDELLA R. y AGUAYO E. (2006): Impacto económico del turismo en el MERCOSUR y Chile (1990-2000), Madrid, AEEADE.

GODED M. y PARACHE M. (2006): El impacto del turismo sobre el desarrollo económico: el caso de Argentina. Madrid, Universidad Complutense de Madrid.

HAVERLAND J.B. (2008): Argentina: Economic, Political and Social Issues. Hauppauge. Nueva York, Nova Science Publishers.

INSTITUTO NACIONAL DE ESTADÍSTICA Y CENSOS DE ARGENTINA (INDEC) (2014): [en línea] URL disponible en: www.indec.mecon.ar/ (Último acceso 16 Mayo 2014).

MINISTERIO DE ECONOMÍA, FOMENTO Y TURISMO (SERNATUR) (2014): Informe Comportamiento y Perfil del Turismo Receptivo 2013. Santiago: Servicio Nacional de Turismo.

MINISTERIO DE TURISMO DE ARGENTINA (2014): Anuario Estadístico de Turismo 2013. Buenos Aires, Ministerio de Turismo [en línea] URL disponible en: http://desarrolloturistico.gob.ar/estadistica/anuarios-estadisticos (Último acceso 03 Mayo 2015)

MINISTERIO DE TURISMO DE ARGENTINA (2014): Indicadores del Turismo. Años 2003-2013. Buenos Aires, Ministerio de Turismo

MINISTERIO DE TURISMO DE ARGENTINA (2013): Anuario Estadístico de Turismo 2012. Buenos Aires [en línea] URL disponible en: http://desarrolloturistico.gob.ar/ estadistica/anuarios-estadisticos (Último acceso 07 Junio 2015)

MINISTERIO DE TURISMO DE BRASIL (2010): Turismo no Brasil 2011 - 2014 - Dados e Fatos. Brasilia. Retrieved 05/27, 2013, from http://www. turismo.gov.br/export/sites/ default/turismo/o_minis terio/publicacoes/downloads_publicacoes/ Turismo_no_Brasil_2011___2014_sem_margem_corte.pdf.

MINISTERIO DE TURISMO DE BRASIL (2013): Anuario Estadístico de Turismo 2013, vol. 40 año base 2012. Brasilia, Ministerio de Turismo [en línea] URL disponible en: http://www.dadosefatos.turismo.gov.br/dadosefatos/anuario (Último acceso 15 junio 2015).

MINISTERIO DE TURISMO DE BRASIL (2014): Anuario Estadístico de Turismo 2014, vol. 41 año base 2013. Brasilia, Ministerio de Turismo [en línea] URL disponible en: http://www.dadosefatos.turismo.gov.br/dadosefatos/anuario (Último acceso $27 \mathrm{de}$ mayo 2015).

MINTEL (2006): Country Reports no.4- Central and South America. London, Mintel International Group.

ORGAZ L., MOLINA L., y CARRASCO C. (2011): «El creciente peso de las economías emergentes en las economías y gobernanzas mundiales». Los países BRICS. Banco de 
España N. ${ }^{0}$ 1101. [en línea] URL disponible en: http://revistas.pucp.edu.pe/index.php/ espacioydesarrollo/article/view/8117 (Último acceso 22 Mayo 2014).

ORGANIZACIÓN MUNDIAL DEL TURISMO (1979): Desglose Regional de las estadísticas del turismo mundial 1973-1977». Madrid, OMT.

ORGANIZACIÓN MUNDIAL DEL TURISMO (1997): Tendencias del mercado turístico. Las Américas 1986-1996. Madrid, OMT.

ORGANIZACIÓN MUNDIAL DEL TURISMO (2011a): Tourism Towards 2030.Global overview. Gyeongju Republic of Korea [en línea] URL disponible en: http://media. unwto.org/sites/all/files/pdf/unwto_2030_ga_2011_korea.pdf (Último acceso 24 Mayo 2014).

ORGANIZACIÓN MUNDIAL DEL TURISMO (2011b): Compendium Tourism Statistics Data 2005-2009 [en línea] URL disponible en: https://pub.unwto.org/epages/Store.sf/ ?ViewAction=DetailSearchProducts $\&$ ObjectID=3650\&Search=SFAllStrings \&Currenc $\mathrm{yID}=$ EUR $\&$ SearchString $=$ compendium $\&$ Category $=3753 \&$ OrderBy $=$ ListPrice (Último acceso 25 Mayo 2014).

ORGANIZACIÓN MUNDIAL DEL TURISMO (2013a): Panorama OMT del turismo internacional, edición 2013. Madrid, OMT [en línea] URL disponible en: http:// mkt.unwto.org/es/publication/panorama-omt-del-turismo-internacional-edicion-2013 (Último acceso 19 Mayo 2014).

ORGANIZACIÓN MUNDIAL DEL TURISMO (2013b): Turismo en las Américas», edición 2013. Madrid, OMT. [en línea] URL disponible en: http://americas.unwto.org/es/ publication/turismo-en-las-americas (Último acceso 18 Mayo 2014).

ORGANIZACIÓN MUNDIAL DEL TURISMO (2013c): El turismo internacional, un motor de la recuperación económica. [en línea] URL disponible en: http://media. unwto.org/es/press-release/2013-12-12/el-turismo-internacional-un-motor-de-la-recuperacion-economica (Último acceso 21 Mayo 2014).

ORGANIZACIÓN MUNDIAL DEL TURISMO (2014a): Tourism 2020 Vision: Americas. Madrid, OMT.

ORGANIZACIÓN MUNDIAL DEL TURISMO (2014b): El turismo internacional supera las expectativas con 52 millones llegadas adicionales en 2013. Madrid, OMT [en línea] URL disponible en: http://media.unwto.org/es/press-release/2014-01-20/el-turismointernacional-supera-las-expectativas-con-52-millones-llegadas-a (Último acceso 19 Mayo 2014) .

ORGANIZACIÓN MUNDIAL DEL TURISMO (2014c): Compendium Tourism Statistics Data 2008-2012 [en línea] URL disponible en: https://pub.unwto.org/epages/Store.sf/ ?ViewAction=DetailSearchProducts $\&$ ObjectID=3650\&Search=SFAllStrings $\&$ Currenc $\mathrm{yID}=\mathrm{EUR} \&$ SearchString=compendium $\&$ Category $=3753 \&$ OrderBy $=$ ListPrice (Último acceso 25Mayo 2014).

PRIDEAUX, B. y CARSON, D. (2010): Drive tourism: trends and emerging markets. London, Routledge.

RIBEIRO, G.L. (2002): «Tropicalismo e europeísmo. modos de representar o Brasil e a Argentina». En: A. Frigerio, \& G. L. Ribeiro (Eds.), Argentinos e brasileiros: Encontros, imagens e estereótipos (pp. 1 -18). Brasil: Vozes Petrópolis. 
RIBEIRO, J.D. y NELSON, S.A. (2004): «La industria del turismo: perspectiva de desarrollo para el Amazonas», Pasos. Revista de Turismo y Patrimonio Cultural, vol. 2, $\mathrm{n}^{\mathrm{o}} 1$, pp. 17-24.

SHARMA, S. (2008): «Emerging International Tourism Markets». New Delhi, Rajat Publications.

SANTANA, G. (2000). «An overview of contemporary tourism development in Brazil», International Journal of Contemporary Hospitality Management. 12/7, pp. 424-430.

SOBRAL, F. PECI, A. y SOUZA, G. (2007): «An analysis of the dynamics of the tourism industry in Brazil: challenges and recommendations», International Journal of Contemporary Hospitality Management, volume 19 (6), pp. 507-512.

UNITED NATIONS DEVELOPMENT PROGRAMME (UNDP) (2013). Human Development Report 2013. The Rise of the South: Human Progress in a Diverse World. New York: UNDP. 